and parasitological processings were all negative. Bone marrow analysis revealed predominance of mature eosinophils without elements of malignant proliferation. Thus, diagnosis of chronic IEP was made. Oral administration of prednisone $(0.7 \mathrm{mg} / \mathrm{kg})$ provided a dramatic clinical improvement in two days. The prednisone dose was gradually reduced at intervals of two weeks and inhaled fluticasone $500 \mathrm{mcg}$ daily was added. After 6 months systemic administration of prednisone was discontinued. However, 7 months later relapse of IEP occured. Again, the boy responded very vell to resumed oral prednisone $(0.35$ $\mathrm{mg} / \mathrm{kg}$ ) which was tapered again over a next 4 months course. Conclusion: The clinical presentation, the course of the disease, the severe eosinophilia of blood and BAL samples, and the absence of other known causes of pulmonary eosinophilia, all these diagnostic clinical elements suggested the diagnosis of chronic IEP. In spite to favourable long-term prognosis further follow-up of the boy is needed because according to modest published clinical experience relapses are always threatening.

\section{THE USE OF NON-INVASIVE MECHANICAL VENTILATION IN THE PATIENT WITH DEFORMITY OF THE THORACIC SPINE - CASE REPORT}

Iva Hižar*, Maja Bosanac, Ivan Pavić, Jadranka Sekelj Fureš. Department of Paediatrics, Children's Hospital Zagreb, Zagreb, Croatia

\subsection{6/archdischild-2021-europaediatrics.428}

Introduction Non-invasive ventilation (NIV) refers to the delivery of mechanical ventilation, without the need for an invasive artificial airway (endotracheal tube or tracheostomy tube). There are different types of interfaces available for use with NIV including: total face mask, oronasal mask, nasal mask, and nasal pillows. The use of NIV has been shown to be an effective in patients with acute or chronic respiratory failure. Scoliosis is a complex three dimensional deformity that leads to impaired chest wall mechanics that prevent normal inflation of the lungs resulting in decreased total lung capacity. Rotation of the chest can produce displacement of the intrathoracic structures and compression of the main bronchi, causing upper airway obstruction. As the disease progresses, due to chronic airway inflammation and and secretion retention, small airways also became obstructed. Over time, ventilation-to-perfusion mismatch leads to chronic respiratory failure. In this case, we emphasize the importance of NIV in the patient with chronic respiratory failure caused by thoracic spine deformities.

Case Presentation A 17-year-old girl has been multidisciplinary followed at our hospital since early childhood due to progressive epileptic encephalopathy that led to severe cognitive impairment. The patient does not have major motor deficits and can walk or move unassisted. Due to recurrent respiratory infections associated with bronchial obstruction, at the age of 14 she underwent diagnostic testing at The Pulmonology, and Allergology division. Allergy tests were negative, and since the patient was uncooperative, pulmonary function tests were not performed. Chest radiograph showed significant progression of the idiopathic thoracolumbar scoliosis. Initially, the patient started therapy with inhaled corticosteroids, and later continued with combination therapy of inhaled steroids and longacting beta2-agonists. Despite therapy, the patient developed severe acute respiratory failure during acute respiratory infections (most often viral infections with secondary bronchopneumonia). In February 2018, she developed severe acute respiratory failure, from which she was slowly recovering and was discharged home after 20 days of hospital treatment, dependent on oxygen. In September 2018 the patient started non-invasive positive pressure ventilation via oronasal mask using Trilogy100 ventilator. Despite severe cognitive impairment, the patient accepted the mask very well, and she continued to use NIV at home only during sleep, an average 6-8 hours per day. With the use of NIV, the patient achieved satisfactory oxygen saturation throughout the day. In the following two years after NIV introduction, the patient did not have any worsening of the respiratory status and her quality of life was significantly improved.

Conclusion Deformities of the thoracic spine can lead to various pulmonary disorders. In patients with chronic respiratory failure, caused by deformity of the thoracic spine, non-invasive mechanical ventilation has proven to be an effective respiratory support.

\section{9 SPONTANEOUS ELIMINATION OF AN INHALED FOREIGN BODY THROUGH THE CHEST WALL}

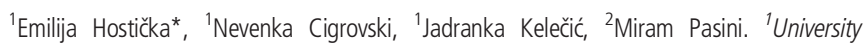
Hospital Centre Zagreb, Department of Pediatrics, Division for Respiratory Diseases, Clinical Immunology and Allergology; ${ }^{2}$ University Hospital Centre Zagreb, Department of Surgery, Division of Pediatric Surgery, Zagreb

\subsection{6/archdischild-2021-europaediatrics.429}

A 20-month-old boy was referred to us from another hospital due to deterioration of the respiratory function during rightsided bronchopneumonia and a suspicion of foreign body aspiration. At the admission, the boy was subfebrile, tachycardic and tachydispnoic, with normal oxygen saturation in the room air, drenched in sweat and with a productive cough. Inflammatory parameters were elevated and X-ray of the lungs showed a suspected inflammatory infiltrate in the right cardiophrenic angle. Bronchoscopy did not show the presence of a foreign body in the respiratory system. During hospitalization, swelling developed in the right infrascapular region with redness, induration and with palpable foreign body in the central part. A surgical incision in the general anesthesia was performed with foreign body extraction. It was a wall barley grass inflorescence (lat. Hordeum murinum). Due to the characteristic structure, wall barley inflorescence tends to move in only one direction. In the case shown, after aspiration, the barley grass spikelets migrated through the tracheobronchial tree, penetrating into pulmonary parenchyma to the periphery of the lung, through the pleural layers, intercostal muscles and finally protruded on the chest wall.

\section{LUNG ABSCESS AS A DIFFERENTIAL DIAGNOSIS OF CHEST PAIN}

Andrea Lidmila Obralić*, Nevenka Cigrovski, Ivan Bambir, Drago Ćaleta, Jadranka Kelečić. University Hospital Center Zagreb, Department of Pediatrics, Department of Clinical Immunology, Respiratory and Allergological Diseases and Rheumatology

\subsection{6/archdischild-2021-europaediatrics.430}

Introduction Chest pain is a common diagnosis in children in puberty and adolescents. Lung abscess is a rare cause of chest pain. It occurs as a result of a lung infection that has led to suppurative necrosis and destruction of the affected lung 
parenchyma. We classify them as primary or secondary, depending on whether there is a pulmonary or systemic disorder. The most common causes of primary abscess are Streptococcus pneumoniae, Staphylococcus aureus and oral bacteria, while the cause of secondary abscess is Pseudomonas aeruginosa, but fungal infections can also be found. Symptoms appear gradually, over several weeks, such as chest pain, cough, fatigue, chills, decreased appetite with weight loss, night sweats, purulent sputum with a sour taste and an unpleasant odor often mixed with blood. After the diagnosis (radiological), taking microbiological materials, empirical broad-spectrum antibiotic therapy is started. Invasive procedures such as surgical open drainage, lobectomy and/or percutaneous drainage are reserved for cases refractory to conservative treatment.

Case Report This is a 14-year-old boy with symptoms of chest pain who appeared the night before the examination in the emergency pediatric clinic. The pain was severe, located in the left side of the chest, exacerbated by inhalation and changes in body position with dyspnea. The analgesic is of lesser intensity. The auscultatory left basal quieter breathing noise is in status. A slight increase in surrogate markers of inflammation was present in the laboratory findings, while radiological processing (X-ray of thoracic organs, ultrasound of the lungs and MSCT of the thorax) verified a vaguely limited nodular shadow about $4 \mathrm{~cm}$ in size at the height of the left hilus. A biopsy of the formation was performed. Cytological analysis of the contents revealed the diagnosis of abscess. He was treated with parenteral antibiotics ceftriaxone and clindamycin for 16 days.

Conclusion Chest pain is one of the common symptoms in an emergency pediatric clinic. The most common pain is benign, musculoskeletal, emotional or idiopathic. Cardiac and pulmonary etiology (ECG, thoracic X-ray, troponin, D-dimers, LDH) should always be ruled out. In this case, we wanted to present a lung abscess as one rare diagnosis that can only be presented by chest pain. It was difficult for the patient to distinguish whether it might be a malignant disease, which we should also consider when treating a patient whose verification of the formation of unclear etiology is verified.

\section{Paediatric Rheumatology}

\section{INCREASED OCCURRENCE OF FAULTY IMMUNOSUPPRESSIVE CELLS IN CHILDREN WITH CHRONIC ARTHRITIS COULD ADVOCATE NEW TREATMENT APPROACHES}

Lovro Lamot*, Alan Šućur, Maša Filipović, Mandica Vidović, Ivana Radoš, Mirta Lamot, Vedran Katavić, Miroslav Harjaček. Sestre milosrdnice University Hospital Center and University of Zagreb School of Medicine

10.1136/archdischild-2021-europaediatrics.431

Juvenile idiopathic arthritis (JIA) is characterized by chronic joint inflammation lasting longer than six weeks, as opposed to the acute reaction in reactive arthritis (ReA) that develops in response to an infection, lasts shorter and usually ends with full resolution of symptoms, indicating different mechanisms responsible for those common forms of arthritis in children. A number of previous studies have indicated the critical role of adaptive immune system cells in the development of many immune-mediated diseases (IMD), including arthritis.
Therefore, the objective of this study was to examine the differences in occurrence of various subsets of lymphoid cells in JIA and ReA patients: regulatory $\mathrm{T}$ (Treg) and regulatory $\mathrm{B}$ (Breg) cells as immunosuppressors, type 3 innate lymphoid cells (ILC3) associated with a wide range of inflammatory disorders by an increase in IL-17 producing T cells and Th17 cells that exhibit plasticity and can be shifted to produce IFN- $\gamma$.

Treg cells, Breg cells, ILC3 and Th17 derived Th1 cells were analyzed in whole blood of ten JIA and six ReA patients by flow cytometry, using directly conjugated monoclonal antibodies. The blood samples were collected during the first visit to Pediatric Rheumatology Clinic in Sestre milosrdnice University Hospital Center in Zagreb, Croatia, while the final diagnosis of JIA or ReA was made three months after. At each visit, juvenile arthritis disease activity score (JADAS-CRP) for each patient was calculated. The median ages of the JIA and ReA patients were 6.41 and 7.22 , respectively.

In patients with JIA, the CD $3+\mathrm{CD} 45+\mathrm{CD} 25+\mathrm{CD} 4$ +CD127-CD28- subpopulation of Treg cells was significantly abundant compared to ReA patients $(\mathrm{P}=0.04)$. No other significant differences in cell subpopulations among different patient groups were observed.

Although Treg frequencies account for only $\sim 5 \%$ of the total CD4+ T-cell population, they have a massive role in the immune response. Particularly,

CD28- Treg cells are characterized by reduced suppression of effector $\mathrm{T}$ cells yielding a pro-inflammatory cytokine profile characteristic for JIA.

Besides, they can be generated in vitro by stimulation of CD28 + Tregs with TNF- $\alpha$, which is raised in JIA. Therefore, the increased occurrence of these cells in JIA patients found in our proof-of-concept study could partially explain the failure of the immunosuppressive mechanisms and the development of the chronic disease in JIA patients. This observation could have a broader clinical significance after confirmation in larger patient cohorts with multiple time points, considering Treg cells have already been shown as a novel therapeutic target in some IMDs.

\section{GUT MICROBIOTA COMPOSITION IN CHILDREN WITH ADVERSE OUTCOMES OF IMMUNE-MEDIATED DISEASE}

I Rados*, M Vidovic, M Cindric, A Hozic, M Harjacek, L Lamot. Sestre milosrdnice University Hospital Center, Zagreb, Croatia

10.1136/archdischild-2021-europaediatrics.432

The perplexing interactions between an organism and it's gut commensal microbiota have both potentiating and detrimental effects on innate and adaptive immunity and consequentially the development of several immune-mediated diseases. Juvenile idiopathic (JIA) and reactive arthritis (ReA) are two adverse outcomes of such disorder in children. This study aimed to assess the differences in the presence of subtypes of Escherichia coli (E coli), one of the most abundant bacteria in the microbiota, in the stool of JIA and ReA patients.

Stool samples of 14 patients with joint swelling were collected during their first visit to Pediatric Rheumatology Clinic in Sestre milosrdnice University Hospital Center in Zagreb, Croatia. Three months later, the diagnosis of JIA was made in seven patients, while the others were classified as ReA. The samples were analyzed by mass spectrometry on nanoLC-Synapt G2 Si instrument. To identify the most abundant E coli 